\title{
Éditorial : Terrains familiers, terrains nouveaux
}

Editorial: Familiar grounds, uncharted territories

\section{Anthony Saber}

\section{OpenEdition}

Journals

Édition électronique

URL : http://journals.openedition.org/asp/5637

DOI : 10.4000/asp.5637

ISSN : 2108-6354

\section{Éditeur}

Groupe d'étude et de recherche en anglais de spécialité

\section{Édition imprimée}

Date de publication : 2 mars 2019

Pagination : 1-2

ISSN : 1246-8185

\section{Référence électronique}

Anthony Saber, «Éditorial : Terrains familiers, terrains nouveaux », ASp [En ligne], 75 | 2019, mis en ligne le 02 mars 2019, consulté le 01 novembre 2020. URL : http://journals.openedition.org/asp/5637 ; DOI : https://doi.org/10.4000/asp.5637

Ce document a été généré automatiquement le 1 novembre 2020.

Tous droits réservés 


\title{
Éditorial : Terrains familiers, terrains nouveaux
}

Editorial: Familiar grounds, uncharted territories

\author{
Anthony Saber
}

1 Le présent numéro nous invite à revisiter et à approfondir des thématiques de recherche traditionnelles en anglais de spécialité (la rédaction technique, illustrée par les instructions techniques et les modes d'emploi), mais aussi à explorer de nouveaux terrains d'investigation: films promotionnels des organismes de santé au RoyaumeUni, première approche de l'anglais vétérinaire envisagé sous un angle diachronique, caractérisation de dictionnaires traitant de la profession policière dans le monde anglophone.

2 Shaeda Isani s'intéresse aux caractéristiques linguistiques et discursives des modes d'emploi et des notices techniques. Comme l'on sait, ces textes procéduraux qui disent «de et comment faire» (Adam 2001) regroupent des productions verbales apparemment hétérogènes (recettes de cuisine, notices de médicaments, instructions d'assemblage). Dans le secteur de l'électroménager, ces textes comprennent des mouvements rhétoriques clairement identifiables (restrictions de garantie par exemple), et reposent sur une posture injonctive (indications sur les choses à faire et à ne pas faire avec un produit par exemple). Emblématique de ce que l'on pourrait appeler la "rédaction technique ", leur style est souvent concis et fondé sur l'asyndète ; il s'astreint à un registre lexical limité (800 mots au total, et une syntaxe simplifiée dans le Caterpillar Fundamental English mis en place par la célèbre entreprise de mécanique américaine pour rédiger ses innombrables documents techniques). Ayant connu leur heure de gloire dans la deuxième moitié $\mathrm{du} \mathrm{XX}^{\mathrm{e}}$ siècle du fait de leur diffusion massive auprès du grand public, les notices techniques et modes d'emploi sont désormais concurrencés par de nouveaux vecteurs d'information technique (diagrammes procéduraux de montage chez Ikea par exemple, ou vidéos explicatives sur YouTube).

3 Caroline Benedetto analyse les vidéos promotionnelles de recrutement diffusées par les organismes de santé britanniques (autorités sanitaires comme le National Health Service, hôpitaux) sur leurs sites Internet institutionnels ou sur les réseaux sociaux. 
S'appuyant sur plus d'un million de salariés au Royaume-Uni, critiqué pour ses dysfonctionnements, le NHS connait des pénuries de personnel et de sérieuses difficultés de recrutement. Il ne faut donc pas méconnaître l'enjeu stratégique de ces films courts, qui présentent l'univers médical britannique sous un angle nettement mélioratif et promotionnel : exaltation de "salariés-ambassadeurs ", héroïsation des personnels soignants (on évoque même les «NHS heroes »), témoignages reconnaissants de patients sauvés par l'action décisive des médecins, courts extraits d'interviews dans lesquelles les soignants décrivent l'intérêt de leur métier et leur passion pour celui-ci. Ces mécanismes d'accroche et de captation visent à imposer un halo rassurant et valorisant autour de l'univers médical britannique par le biais de représentations positives. De manière intéressante, les aspects spécialisés (procédures de traitement, équipements, terminologie technique) sont escamotés dans ces vidéos, fondées sur des techniques de marketing largement éprouvées pour assurer la promotion de biens et services auprès du grand public.

Muriel Conan défriche un domaine pour l'heure très peu étudié en anglais de spécialité, à savoir l'anglais vétérinaire. De nature diachronique, son étude s'appuie sur un corpus d'articles publiés dans la revue professionnelle In Practice, bien connue des vétérinaires britanniques. L'analyse des sommaires de cette publication et du vocabulaire-clé utilisé dans les articles montre l'évolution des préoccupations professionnelles à trente ans d'intervalle (1987-2017). Alors que les thématiques évoquées en 1987 touchaient essentiellement aux soins vétérinaires eux-mêmes, de nouveaux sujets s'imposent dans la période récente : gestion administrative du cabinet vétérinaire, considérations éthiques, prise en charge de la douleur chez l'animal, désormais considéré comme un "animal-patient». Cet élargissement thématique illustre la complexification croissante du métier vétérinaire, qui doit intégrer désormais, au-delà des actes et des interventions de nature strictement médicale, des dimensions gestionnaires, juridiques et éthiques.

Audrey Cartron se penche quant à elle sur plusieurs dictionnaires ou encyclopédies traitant de l'univers policier. Elle propose plusieurs paramètres de caractérisation : ces publications, rédigées par des acteurs du domaine, mais aussi par des chercheurs, sontelles de nature essentiellement terminographique, ou ont-elles des intentions encyclopédiques? Ont-elles une visée internationale, ou sont-elles ciblées sur l'aire anglophone? Se prévalent-elles d'une objectivité revendiquée, ou adoptent-elles une posture plus axiologique? Se préoccupent-elles surtout des concepts et savoir-faire policiers dont le sens ne serait pas transparent pour le profane, ou englobent-elles également des notions dont la compréhension est plus immédiatement accessible? Quel est le lectorat visé ? Quel est le degré de permanence des contenus décrits, quelles mises à jour sont nécessaires pour prendre en compte l'évolution constante des méthodes et savoirs policiers? Ceux-ci sont de nature fondamentalement interdisciplinaire, se situant au carrefour des techniques d'enquête, des aspects juridiques et judiciaires, de la médecine légale, de la psychologie individuelle et sociale, de la criminologie, voire de certaines sciences (biologie pour ce qui touche aux analyses ADN par exemple). La « dictionnairisation » des notions consignées et codifiées dans les publications analysées par Audrey Cartron illustre ainsi, malgré le caractère hétéroclite des sujets traités, la cristallisation de la profession policière en doctrines constituées. 
6 Un compte-rendu d'expérience pédagogique rédigé par Nadeera Rajapakse et deux recensions par Adeline Terry et Rita Temermann vous sont également proposés dans ce numéro 75.

\section{BIBLIOGRAPHIE}

ADAM, Jean-Michel. 2001. « Types de textes ou genres de discours. Comment classer les textes qui disent « de et comment faire ». Langages 141, « Les discours procéduraux », 10-27.

\section{AUTEUR}

\section{ANTHONY SABER}

Rédacteur en chef. anthony.saber@ens-paris-saclay.fr 